\title{
Facilitating Narrative Change in Career Construction Counseling
}

\author{
Abstract \\ Career Construction Counseling fosters client change by evoking and \\ elaborating innovative moments in client narratives. In this article, we \\ describe four types of dialogues that counselors may use to prompt \\ narrative novelty and foster client change: (a) identify and evaluate the \\ effects of innovative moments, (b) highlight contrasting self-positions, (c) \\ ask about changes achieved, (d) promote a metaperspective on change. \\ Vignettes from a case are used to illustrate how to use IM markers as a \\ heuristic guide for when to engage in these four types of dialogues.
}

Keywords: career construction counseling, innovative moments, client change, evoking change, elaborating change

Fostering change is the main aim of any psychological practice including career construction counseling (CCC; Savickas, 2019). Exploratory research on the process of CCC (Cardoso et al., 2014a, 2014b, 2016), grounded in innovative moments model (IMM; Gonçalves et al., 2009), has produced a heuristic model that describes how clients change in good outcome cases (Cardoso et al., 2019). The parallel between the progression of innovative moments (IMs) and counseling tasks in the three phases of $\mathrm{CCC}$ has explicated the role of counselor interventions in prompting client change during narrative career counseling. This article describes four types of dialogues that counselors may use to evoke and elaborate narrative innovation that indicates client change during CCC.

CCC Effectiveness

$\mathrm{CCC}$ is a career counseling discourse that takes a narrative perspective on psychological functioning, one that views an individual's stories as a means to organize personal experiences 